\title{
The physical investigation on the transmission coefficient of Pile-supported vertical wall breakwaters
}

\author{
KeXinZhang ${ }^{1, a}$, JinSongGui ${ }^{1, b,}{ }^{*}$ andDongXuWang ${ }^{1, c}$
}

College of Ocean and Civil Engineering, Dalian Ocean University,Dalian Liaoning116023,China

aEmail:949842426@qq.com

bEmail:guijs@163.com(*corresponding author)

'Email:21140216@qq.com

Key words: transmission coefficient,Pile-supported vertical wall breakwaters, model test

Abstract:Through the physical model test of Pile-supported vertical wall breakwaters, the influence of various elements of wave on the transmission coefficient under the regular wave is analyzed, and the relationship curve is plotted through the non- Analysis of the relationship between them for easy reference to similar projects.

\section{Introduction}

The application of Pile-supported vertical wall breakwater are widely used around the world for the construction of harbours and for shore protection works. Compared with the traditional breakwater.It can make use of the wave blocking structure to reduce the impact of waves on the construction by causing the wave turbulence and convection; it also reflects part of the wave energy ${ }^{[1]}$ However, it does not prevent the deposition of sediment into the river, which is generally applicable to situations with larger water depth, smaller wave height and less sediment ${ }^{[2]}$. The transmission coefficient is the main factor for the determination of the wave abilities of pier-pier breakwater.

For the study of transmission coefficient, foreign countries began in the middle of the 20th century, UrsellF gives the exact solution of the wave projection coefficient in the case of infinite water depth upright sheet. Wiegel gives an approximate analytic solution of the transmission coefficient for a finite water depth without surging assuming that the transmitted wave energy equals the incident wave energy under the baffle. Our country has also done a lot of research on this. In 1986, on the Wiegel formula, DahongQiu proposed a diffraction phenomenon, that is, part of the reflected energy is directly reentered, and some energy is diffracted from the bottom of the baffle to the baffle Back; Hehai University Yixin Yan through the multi-layer baffle pier permeable breakwater research, the permeability coefficient obtained through the different permeability and baffle settings greater influence. 


\section{Experimental equipment and experimental setup}

Laboratory equipment

Experiment flume

The experiment was carried out in the wave tank of Key Laboratory of Coastal Engineering of Dalian Ocean University in Liaoning Province. Effective use of the sink size $40 \mathrm{~m}$ long, $0.7 \mathrm{~m}$ wide and $1.0 \mathrm{~m}$ deep, can accommodate a maximum depth of $0.7 \mathrm{~m}$, one end of the tank equipped with a motor to serve irregular waves wave generator $(1.0 \mathrm{~m} \times 0.7 \mathrm{~m})$, the simulation can be generated in the cycle $0.5 \sim 5 \mathrm{~s}$ regular waves, irregular waves and other waves. The back of the wave machine and the tail of the tank are equipped with energy dissipation devices such as vertical energy dissipation grids and energy dissipation overhead ramps to avoid the reflection of waves.

Wave measurement

The wave measurement uses the DS30 wave height water level measuring instrument developed and produced by Tianjin Academy of Water Sciences. The instrument has built-in analog-to-digital converter and patrolled to collect the data of each channel. The single point sampling interval is $0.0015 \mathrm{~s}$ (about $666 \mathrm{~Hz}$ ) The minimum sampling interval is 0.01 seconds $(100 \mathrm{HZ}$ ); the minimum sampling interval for 128 channels is 0.02 seconds $(50 \mathrm{~Hz})$. The system can simultaneously measure multi-point wave surface process and data analysis, has been applied in a number of physical model test, accurate and credible. Calibration before each test, calibration linearity greater than 0.999 .

\section{Data collection}

Wave surface data acquisition using China Institute of Water Conservancy and Hydropower Research DJ800 monitoring system, which is composed of computers, multi-functional monitor and a variety of sensors consisting of data acquisition and data processing system. The data acquisition is touring. It takes about $400 \mu \mathrm{s}$ to obtain the data of one measurement point (including the selected channel number, analog-digital conversion, data transfer time, etc.). Therefore, the sampling frequency decreases as the number of measurement points increases.

\section{Experimental setup}

The longitudinal section of the wave flume in the experiment and the layout of the relevant model are shown in Fig.1, a total of four wave height meters were arranged. Before the breakwater, the wave probe 1 and 2 were used to measure the reflected wave height.

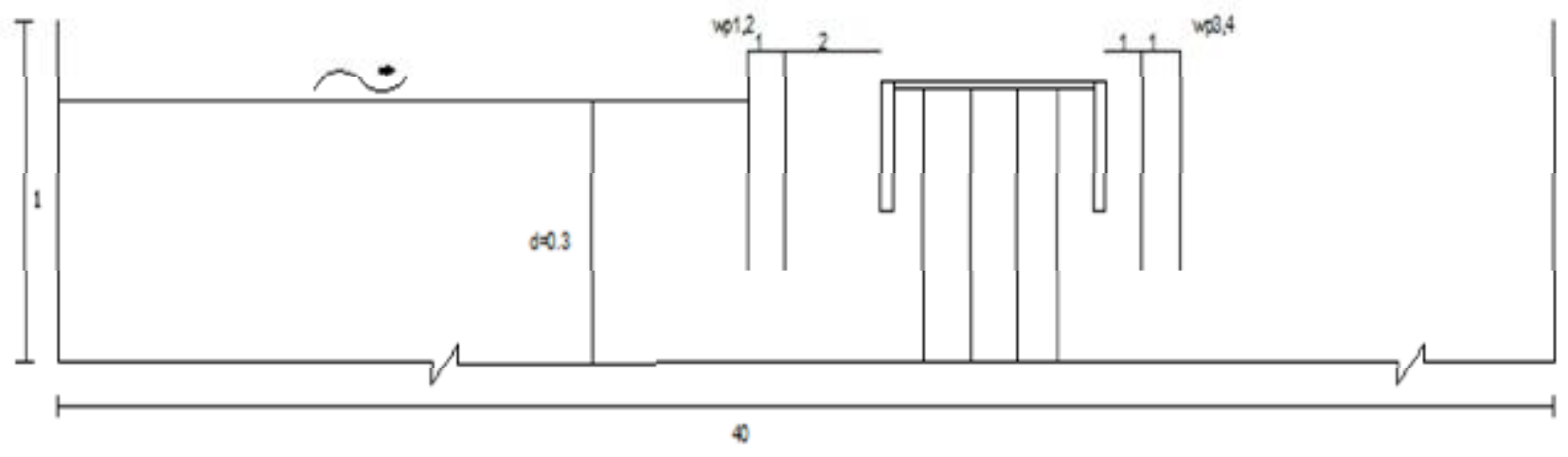

Fig.1.Wave flume profile and model layout [wp=wave probe, unit:m] 


\section{Experimental parameters}

In this experiment to maintain the bank width $B=70 \mathrm{~cm}$ and water depth $d=30 \mathrm{~cm}$ are unchanged, the wave elements used in the test in Table 1. Before and after the baffle into the water depth shown in Table 2 .

Table.1. Wave property[Unit: $\mathrm{cm}]$

\begin{tabular}{|c|c|c|c|c|}
\hline Wave length & $\mathrm{L} / \mathrm{cm}$ & Wave period & $\mathrm{T} / \mathrm{s}$ & Wave height \\
\hline 137 & & 1 & & $4 / 6 / 8 / 10 / 13$ \\
\hline 177 & & 1.2 & & $4 / 6 / 8 / 10 / 13$ \\
\hline 289 & & 1.8 & & $4 / 6 / 8 / 10 / 13$ \\
\hline
\end{tabular}

Table.2.Front and rear baffle into the water depth[Unit: $\mathrm{cm}]$

\begin{tabular}{cc}
\hline front barrier[cm] & rear barrier[cm] \\
2.5 & $2.5 / 5 / 7.5 / 10$ \\
5 & $5 / 7.5 / 10$ \\
7.5 & $7.5 / 10$ \\
10 & 10 \\
\hline
\end{tabular}

\section{Experimental results and discussion}

Transmission effective wave height $\mathrm{Ht}$ and incident effective wave height $\mathrm{Hi}$ is the transmission coefficient. Which H1/3 is usually considered to be effective wave height.According to the previous study on pile baffle through the air-through can know that the transmission coefficient is mainly affected by wave height, cycle, baffle into the water depth and many other factors.In order to better apply the value in the project, it is expressed in a dimensionless manner:

$$
K_{t}=\frac{H_{t}}{H_{i}}=f\left(\frac{B}{L}, \frac{\Delta}{H}\right)
$$

\section{$B / L$ effect on the transmission coefficient}

The width of the embankment is the ratio of the width $\mathrm{B}$ of the embankment breakwater to the wavelength $\mathrm{L}$ of the incident wave.It can be seen from the expression that the width relative to the embankment is mainly caused by the action of breakwaters and waves in the unit wavelength Of the energy dissipation to reduce the transmission coefficient, with the increase of B / L, the greater the breakwater and the wave interaction, the more energy consumption, the smaller the transmission coefficient, $B / L$ on the transmission coefficient shown in Fig 3,4 . 


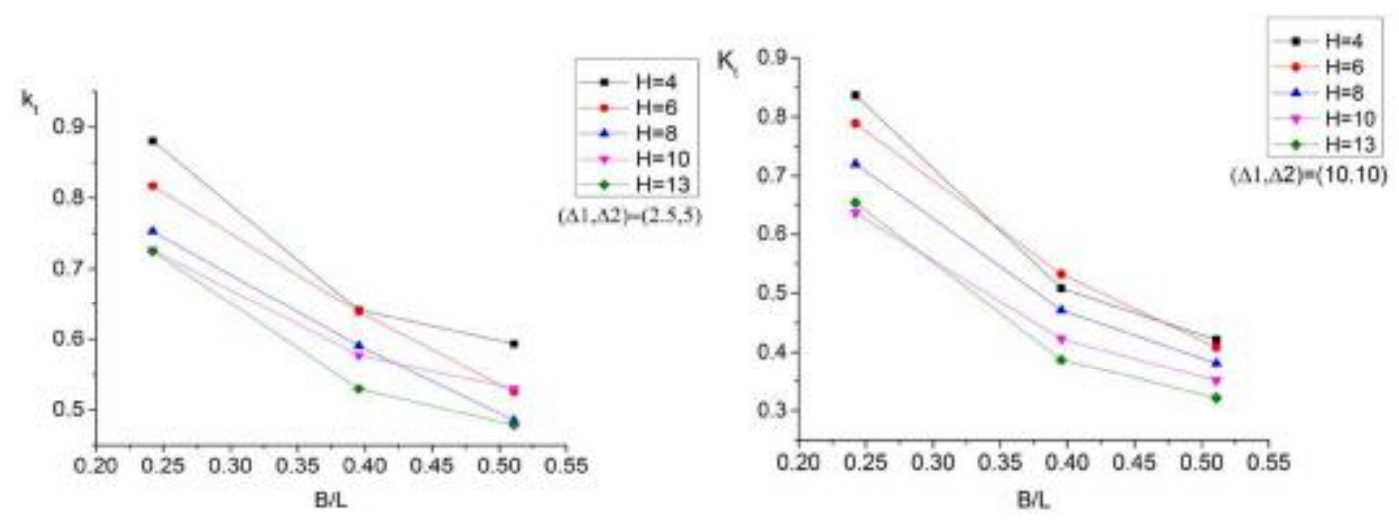

Fig.3,4. effect on the transmission coefficient[unit:cm]

From Fig. 3 and Fig. 4, we can see that with the increase of the width $B / L$ of the embankment, the transmission coefficient decreases obviously, indicating that the influence of the width of the embankment on the transmission coefficient is larger. When $0.23 \leq B / L \leq 0.4$, the change of transmission coefficient is steeper than that of $0.4 \leq B / L \leq 0.53$, indicating that in practical engineering, the width of dike should be increased appropriately, and when it reaches a certain value, The width of the embankment is uneconomical.

$\Delta / H$ effect on the transmission coefficient

The main function of pile-type baffle piercing breakwater is to reduce the transmission coefficient through the interaction of the baffle with the incident wave. With the variation of baffle penetration depth and wave height ratio, the transmission coefficient will also show a certain rule. $\Delta / h$ on the transmission coefficient as shown in Fig 5,6,7.

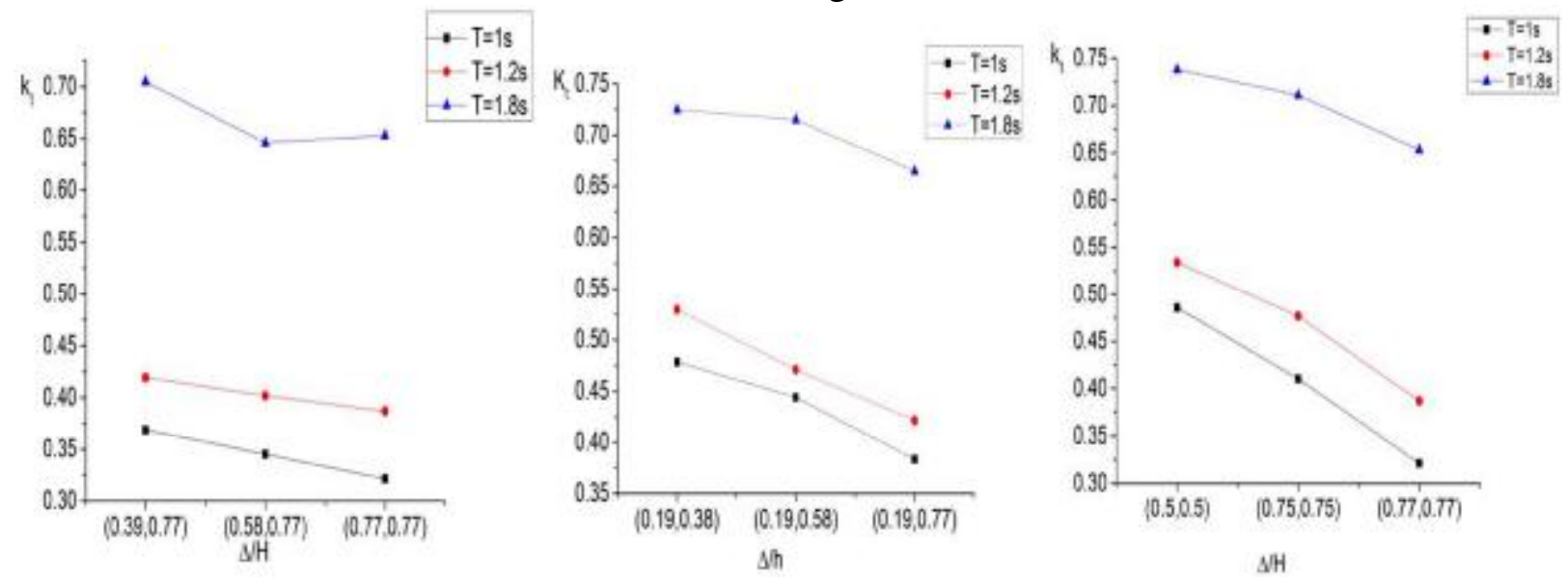

Fig.5,6,7. $\Delta / \mathrm{H}$ effect on the transmission coefficient

It can be seen from Fig. 5, 6 and 7 that as the ratio increases, the transmission coefficient decreases. As can be seen from Figure 3, in the back of the ratio of a certain time, increasing the ratio of the front, the transmission coefficient decreases is not obvious. As can be seen from Figure 4 , when the front ratio is fixed, the ratio of the back increases, and the transmission coefficient decreases a lot. As can be seen from Figure 5, in the same ratio of before and after the case, increasing the ratio, the effect is much better.

\section{Conclusion:}

Through the wave flume experiment, it is found that the transmission coefficient is mainly related to the width of the embankment under the regular wave. Within a certain range, the relative 
increase of the width of the embankment will reduce the transmission coefficient a lot, Width does not have a great effect, and not economical and reasonable. The ratio of the baffle to the wave height will have a greater effect on the transmission coefficient when both front and back are the same. Relatively increasing the ratio of the front of the transmission coefficient is not very effective in reducing the effect; on the contrary to increase the latter ratio will greatly reduce the transmission coefficient. In general, because of the influence factors of transmission coefficient, it should be analyzed through physical model in engineering practice.

\section{Reference}

[1] WeBingFeng,WenLlinDing and XiaoHongYe, Research on Penetrating Wave Characteristics of Perforated Breakwater with Pile Foundation:Waterway Engineering 62-67(2014).

[2] YuliangLiu,kunJing andHaiFengWang Influence of Wavelength on Permeability of Piles Pierced Breakwater:Waterway Engineering70-74(2014).

[3] S. Neelamani andM. Vedagiri, Wave interaction with partially immersed twin vertical barriers:Ocean Engineering 29 215-238(2002)

[4] DaHongQiu and XueGengWang, Theoretical Analysis of Deepwater Thin Plate Breakwater:Waterway Engineering8-12(1986).

[5] YiXin Yan,JinHaiZheng and XiaochuanZeng, Experimental Research on Wave Characteristics of Permeable Breakwater with Pile Foundation:Ocean engineering67-74(1998)

[6] ZhiXiongMa,Experimental Research on Wave Breaking Performance of Breakwater Through Breakwater:Nanjing Water Institute 1-6(2002)

[7] DanJunYu,WeiBingFeng and YanBoLi,Test and Calculation of Horizontal Wave Pressure of Pierced Double Wall Breakthrough Breakwater:Waterway Engineering19-24(2015)

[8] Bennet G.S., Melver,P., Smallman,J.V.:A mathematical model of a slotted wave screen breakwater:Coastal Engineering 234-249.(1993). 